\title{
Interview with Dr. Domingo Martin on "Temporomandibular Joint, Functional Occlusion and Excellence in Orthodontics"
}

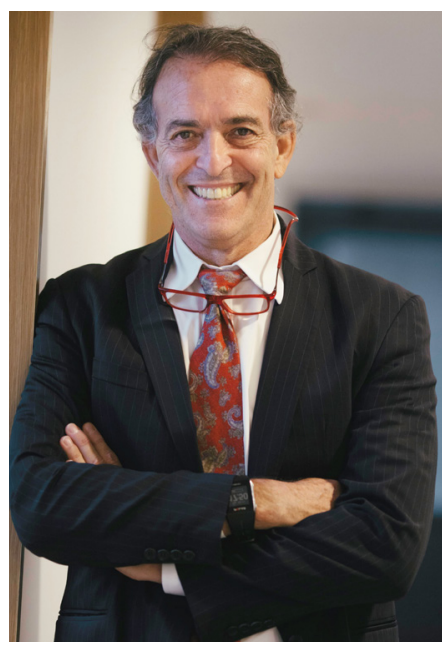

TJO: In orthodontics, our objective is to establish an ideal occlusion with good facial esthetics and stability. Is there something missing?

Dr. Martin: There is something missing but it is because people don't have a clear understanding of what a good occlusion is. It is easy to say an ideal occlusion. I can still remember a well-known orthodontist used to always say "We gave the patient a good functional occlusion." And everyone uses the word "functional occlusion". But not everyone understands what a good functional occlusion is. We wrote an article about stability and in the article, I made the statement "we need to change the definition of occlusion". It is not me who says this. It is the president of the American Academy of Restorative Dentistry. He says, "If we only look at teeth, and if we only think that occlusion is teeth, we are never going to understand occlusion fully." What is an ideal occlusion? Well, the ideal occlusion is an occlusion that is in harmony with the stable condylar position. If we only look at teeth, we can say that they fit nicely but we cannot say that is an ideal occlusion. Let me see how you bite, how you chew, if you have the correct arc of closure and all of your teeth hit simultaneously bilaterally with the condyles seated. This is something that Jeff Okeson says. My definition of an ideal occlusion would be orthopedic stability. I think we spend too much time talking about occlusion, but occlusion is not really explaining the whole situation. When we use the word "occlusion" we only talk about teeth. When you talk about "orthopedic stability", you talk about teeth, joints, arc of closure, and chewing pattern. I agree that the objective should be facial esthetics but also orthopedic stability.

TJO: Why is orthopedic stability so important in orthodontics?

Dr. Martin: I guess it is because I have grey hair. The older I get, the more I understand how important orthopedic stability is. I am now being able to see my patients 20-30 years posttreatment. What do I see? I see that the cases are very stable. When I say stable, I mean no tooth wear, no TMJ symptoms, and very little crowding. I don't care about minor crowding, I think we should not be obsessed with $1.5-2 \mathrm{~mm}$ of lower incisor crowding. There is nothing stable in the universe, everthing moves. Why should teeth not move? However, we don't want 6-8 mm of crowding. So, when you achieve orthopedic stability you also achieve the correct arc of closure. And this is so important. When the patient opens and closes, there should be nothing getting in the way of the mandible during mastication, chewing or autorotation. The teeth should hit perfectly and evenly. This is the only way that you can have a good functional occlusion where the canines and the incisors disocclude the posterior teeth. For this to happen you have to have a stable condylar position. The minute that you don't have orthopedic stability, you don't have functional occlusion. And then, we see tooth wear, muscle symptoms, sometimes TMJ symptoms, and much more lower incisor crowding. I think that the lower incisors are not receiving the forces along their long axis. They are receiving lateral and oblique forces because of the mandible not moving correctly when we don't have the correct arc of closure. So, this is why for me orthopedic stability is so important. Because it is all about longevity. It makes me upset seeing a mother who says "Doctor, I had orthodontics when I was young.." When I look at her teeth, I am ashamed. If this is what orthodontics is going to give you 25 years from now, well, something is wrong. I don't mind a little bit of tooth wear, or maybe a little bit of lower incisor crowding. But it is not what I see. Usually, when I retreat many patients who had orthodontic treatment, the first thing I notice is the orthopedic instability. We are paying a price for orthopedic instability and yet we still do not give it importance. I heard recently a speaker saying that tooth wear was totally normal, and we should wear our teeth just as our ancestors. I don't accept this. 


\section{TJO: Do you use fixed retainers?}

Dr. Martin: I do use fixed retainers. But I usually make them out 1-2 years later. And I tell the patients and the parents that there is going to be a little bit of tooth movement. I don't want to be responsible for fixed retention. I see lots of unwanted side effects with fixed retainers. We have to be careful and take full responsibility for fixed retainers. After all it's us who placed them.

TJO: In the literature, there is no consensus regarding the relationship between occlusion and the temporomandibular disorders. How do you comment on the potential role of the malocclusion in the onset of the temporomandibular disorders?

Dr. Martin: Let's start from two very well respected doctors, Dr. Jeff Okeson and Dr. James McKee. Dr.Jeff Okeson says that it is difficult to find a relationship between the occlusion and the temporomandibular joint if we continue to look at static occlusion. We have to realize even though there is lots of literature, they are still looking at static occlusions. I can look in a patient's mouth looking like a beautiful Class I, but he has symptoms. We place a splint, and it is no longer Class I. All the studies are being done with Class I occlusions as ideal can be a false position. This is why Dr.McKee talks about changing the definition of occlusion. He says "if we change the definition of the occlusion, it will probably come a time where we will find a relationship between the occlusion and TMD." But if we only look at the teeth, it will be difficult to find a relationship. I have spoken with many specialists, some agree, and some don't. You can not trust what you see in the mouth, because it is not what really exists. I saw a patient last year. He had the worst tooth abrasions, and he was only twelve years old. Both joints were on the eminence. How many people are looking at joints in 12 years old children? When I see that amount of tooth wear, the first thing that I think is something is wrong in the system. It is not about stress, he plays football all day, he is on the bike, he is happy and doesn't worry about anything. Why does he have tooth wear? This is a beautiful example of orthopedic instability. It is probably related to tooth wear. I say probably, because the day that I finish his treatment and restore his teeth, I will have to see the patient five years later at least to see if he stops wearing his teeth. But I have done it enough times so I know he will stop wearing his teeth. I respect the literature but when it comes to occlusion, the problem is the definition of the word occlusion. As long as we only talk about teeth, we are never going to find the relationship. In fact, what Dr.McKee says "much of the confusion that we see in the literature relating occlusion to TMD is because of the definition of occlusion". We need to bring the researchers and the clinicians together. We have been saying this for years, but we never do it. Many of the researchers are surrounded by the clinicians who already think that the teeth have nothing to do with TMJ. So, it is not evidence based but bias based. These are not my words but the words of Dr.Vanarsdall. They already know what they believe and that is what they are going to research. I think we need to do more research and that is what I am doing right now.

TJO: Dr. Martin, this is a very good suggestion for the researchers to come together with the clinicians.
Dr. Martin: Yes, I have many university students doing research in my office. A group from Budapest is looking at the relationship between vertical control and facial esthetics. Another researcher is evaluating the relationship between vertical control and the airway. As you can see, I am trying to bring evidence to what we do. However, in the world of TMJ, it is not so simple. There are so many variables.

TJO: Dr.Roth says "Don't believe what you see in mouth!". What are your essential diagnostic tools for treatment planning?

Dr. Martin: Let's not forget that we were not born to have beautiful teeth. That is our invention. We were born to have teeth to be able to chew and to be able to survive. However, in the 1920s and 1930s, Hollywood decided that the actors should have beautiful teeth. That is when the orthodontic movement began, and their goals were mainly esthetics. But the main goal of teeth is to survive, even today. That means that the teeth will always reach the maximum intercuspation in spite of tooth wear and condyle position to be able to chew. It is not easy to chew on 2 or 3 molars. The patients who have open bite will tell you. They don't chew, they swallow. There is no doubt that if these patients who have open bites could bring their mandible forward and bring the teeth together, they would do it. But they cannot. However, many patients move the mandible forward unconsciously, out of the condyle position, to bring the teeth into maximum intercuspation. So, why does Dr.Roth say, "Don't believe what you see in the mouth." ? Very simple, because of the definition of occlusion. If you think that occlusion should be a condyle position and a dental position, you have to look at the teeth, but you also have to check the joint position. "Does the patient bite correctly?". You should always ask yourself that question. When I see ideal teeth, I want to see how the patient really bites. When the patient bites really well, I say this is good occlusion and this is orthopedic stability. However, when I see a good dentition, and take the mandible and all of a sudden, I realize that there is a contact at the back, I know that the patient is now autorotating to avoid the contact, I cannot trust what I see in the mouth. Unfortunately, this happens in a large percentage of patients who have problems. These problems are almost always associated to an unstable occlusion and orthopedic instability. Don't trust what you see in the mouth, because it has nothing to do with the mounted model. Sometimes it is not easy to find the first contact because of the muscle splinting taking the mandible to maximum intercuspation. It is difficult to change this muscle ingram. This is why we use splints which relax the muscles and move the condyles to a stable position. I don't know where that position is. No one knows and it doesn't matter. All that matters, is if the patient is easy to manipulate and we have our arc of closure. Some symptomatic patient become asymptomatic. However now we have a new dental position. We use splints because we want to diagnose. There is no doubt that the splints help us to resolve many symptoms such as muscle pain, headaches, shoulder and neck pain, but it is actually a diagnostic tool. There is more to a splint that just to resolve symptoms, the advantages of using a splint is because now you know exactly what you need to do to maintain the patient asymptomatic. The splint is telling you "don't believe what you see in the mouth, this is now the real occlusion and 
you have your job to do". There is no doubt that the skeletal anchorage has changed our life tremendously. In the past, it wasn't easy to solve these problems. We had to extract more teeth and do more surgery. Now, it is so much easier. I am not afraid to put a splint in a patient's mouth. Dr. Kazumi lkeda used to call it "the hidden open bite". When you splint the patient, you are probably going to get an open bite, which was already there but it was hiding from you.

TJO: Apart from the splints, what other diagnostic tools do you use?

Dr. Martin: I cannot imagine my office today without CBCTs. In fact, if it wasn't because of the ethical and the radiation, I would probably take CBCTs on $100 \%$ of my patients. I am very cautious. I don't want to take CBCT of children because I don't need to on a routine basis, but when I need to, there is no doubt I will take a CBCT. CBCT is an important tool in my diagnostic process when there is orthopedic instability. When there is orthopedic instability, the joints and the teeth don't speak the same language. The CBCT will tell us: do we have a normal joint? Do we have a joint suffering from degenerative joint disease? Has the patient suffered from degenerative joint disease in the past? Is it symmetrical or asymmetrical? Are the joints well positioned in the fossa? It is not easy to make this decision because there are variations. But the variations should be within the norms. You can not say it is out of the fossa because it is slightly down and back. But you can say that there is tendency for that condyle to be down and back. However, in many instances, it is so far out of the fossa that you know that the condyle is not in the fossa. Now you know that there is an orthopedic instability situation because the joints are not in the correct position. Don't forget, the teeth take the joints out of the fossa. The joint is where it is due to the teeth, they are responsible for this position. What is happening at the tooth level that makes these joints go back. This is the diagnostic process that you have to look at. Do I take MRIs? I barely take MRIs. Because I don't need them. By looking at CBCT and by doing my joint analysis, I can more or less tell if the disc is displaced with or without reduction or if the patient has no disc. Everytime there is degenerative joint disease, the discs are gone. When the disc is totally displaced, as orthodontists we have a very important job to do. We have to stabilize the joint on the posterior ligament. The posterior ligament is going to be the pseudo disc. We have to take care of the posterior ligament very well, because this is what is going to be able to keep the mandible in the correct stable position and the patient asymptomatic. We also look at MRIs and mountings of course. The mountings will tell us if there is MIC-OC discrepancy. Then we look at the CBCTs and do our joint analysis. We also look at the skeletal pattern. Dolichofacial patients have more problems than normal patients.

TJO: Are you advocating the mounting of the study models?

Dr. Martin: I always say that you can do very good orthodontics without mounting models. However, you are going to miss some patients. You think that some patients have a good occlusion, and you take the mounted models, and you say "wow". It is not easy to see by just looking in the mouth and white handheld models do not always give you the entire information you need. How do you reach that level of perfection where you know exactly who you should mount and who you should not mount? It is very difficult. However, Dr.Roth mounted all his patients, there was a time where he was choosing which patients to mount and which not to mount until he realized that he had made many mistakes. Therefore, he one day decided just to mount all patients to make sure he did not make mistakes. Even in today's digital world and CBCTs, it is still important to mount models. Without any doubt the future will be digital mounted models.

TJO: Do you prefer digital mounting instead of analog systems?

Dr. Martin: I don't prefer it because I was born with models and articulator in my hands. I will always miss it, but I am sure the new generations are not going to miss it and although I will miss it does not mean that I will not start using this technology. On the contrary, I am an avid user of digital technology. In the digital world, you can now incorporate 4D movements with the scanned models or teeth, use CBCT and with the new ModJaw tracking system, we can incorporate the correct center of rotation which will revolutionize today's world of dentistry. Just because you have an articulator on the screen and you move the articulator, does not mean that you have the correct arc of closure. That's why I don't care for these programs that use arbitrary center of rotation, they are nice but they are not precise. We are more precise because we are incorporating the center of rotation, the correct arc of closure with the scanned dentition all at the same time. In fact, to tell you the truth, we don't take impressions, face bow transfers and mounted models anymore.

TJO: How do you incorporate the movements with the digital models?

\section{Dr. Martin: We use different software programs.}

TJO: According to your treatment philosophy, the correct 3D positioning of the posterior teeth is a very important step. Could you explain why we need to pay attention to the vertical control of the molars?

Dr. Martin: It is all about the arc of closure. The problems always arise at the posterior teeth. And it is almost always a transverse problem. Dr.Vanarsdall used to talk about this so often. He wasn't thinking about the second molars. But Dr.Vanarsdall gave us reasons why the transverse dimension was so important and why we needed to expand. Even though there wasn't a crossbite, we still needed to expand. What I realized over the years is that the second molars which are so important in the arc of closure are usually the reason why there is false position of the condyle and you cannot trust what you see in the mouth. It is mainly transverse in a large percentage of the patients. Both dental and skeletal transverse problems should be corrected. Sometimes you need to correct the torque. However, when you incorporate the skeletal pattern, now you have to change the torque and intrude the molars because of the posterior rotation of the mandible. The second molar is very important. We still see the JCO's surveys that a large percentage of orthodontists are still not banding the sec- 
ond molars. That is very sad because we are giving the patients esthetics but we are not resolving the functional problem. And some patients are going to suffer because of that. I resolve most of these problems by treating the second molars and it works. Patients are asymptomatic, they have no tooth wear, we do full mouth reconstructions with composite and they are stable.

TJO: There is a changing trend about extraction treatment toward nonextraction by the use of TADs and expansion. What is your opinion?

Dr. Martin: There is no doubt that the number of extraction cases have come down a large amount. I have never cared about distalizing molars, because it wasn't an ideal treatment or solution for avoiding extractions since it was very difficult to move the molars en masse. But today, with TADs and especially palatal skeletal anchorage, we can move these molars almost en masse. This is of course avoiding lots of extractions. In the past I would never expand to avoid extractions but today we are avoiding extractions by expansion because we can expand more than before due to palatal anchorage. So, there is no doubt we do less extractions. But I just wrote an article about stability and I wrote about the importance of taking a mounted model before you take off the appliances. It is very nice to avoid extractions, but you have to ask yourself "Did I achieve all the goals?". Because our goal should not be nonextraction. Are we treating too many patients nonextraction because of TADs and because of expansion and not achieving our goals? Then, this is a problem.

TJO: What are the key factors for stability?

Dr. Martin: The number one factor for stability in my opinion is occlusion. Teeth need to receive occlusal forces along their long axis. However, this should happen with the condyles seated, with the correct arc of closure, bilateral simultaneous contacts on all teeth. This is of course orthopedic stability. Nothing is isolated, they are all interrelated. So, occlusal forces are the most important factors for stability. When teeth receive occlusal forces along their long axis, they are going to be more stable. Another factor for stability is tooth eruption. This is why I extract many deciduous teeth. Because I want the teeth to erupt in the mouth with no rotations. This is another very important factor because a minute a tooth erupts rotated, it has memory. When I see that I have lack of space for the lateral incisors, I will extract deciduous canines immediately because I want the lateral incisors to erupt perfectly aligned in the mouth. This is another very important factor for stability, to have the teeth erupt in the correct position in the dental arch.

\section{TJO: How would you define the excellence in orthodontics?}

Dr. Martin: If you achieve all the goals in all your patients, you are the best orthodontist in the world. And we all know it is not easy to achieve all the goals in all patients. Facial esthetics is so important and everything you do to improve facial esthetics is going to be an excellent treatment. Sometimes you have to go for the surgery. But today with skeletal anchorage, we are seeing lots of incredible skeletal changes. Dental esthetics is another factor for excellence but is not always in our hands. We have small, abraided or colored teeth. In order to have excellence, you need a good prosthodontist in your team to be able to finish your cases. Then, you need orthopedic stability, longevity of the teeth and no abrasion. If you have good facial and dental esthetics, functional occlusion, periodontal health and stability, that is excellence in orthodontics. I think that excellence is an attitude. You have to want the excellence, you have to convince your patients that excellence is important for them and for you. And then, you have to go for it. But you also need to know your limitations.

TJO: Thank you very much for this informative interview. 